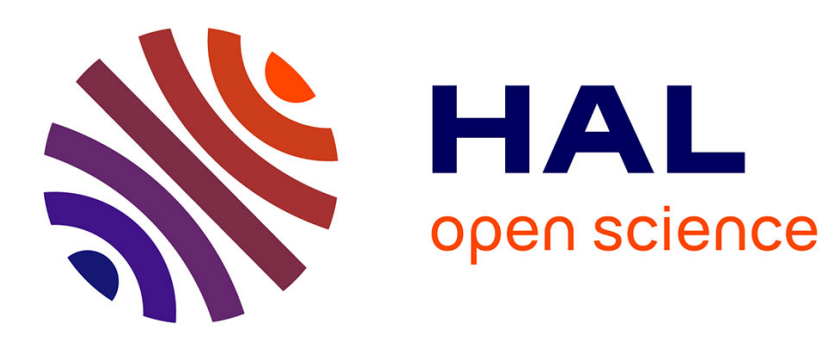

\title{
De l'histoire des concepts à l'histoire linguistique des usages conceptuels

\author{
Jacques Guilhaumou
}

\section{To cite this version:}

Jacques Guilhaumou. De l'histoire des concepts à l'histoire linguistique des usages conceptuels. Genèses. Sciences sociales et histoire, 2000, 2000/1 (38), pp.105-118. 10.3917/gen.038.0105 . halshs02525189

\section{HAL Id: halshs-02525189 \\ https://shs.hal.science/halshs-02525189}

Submitted on 30 Mar 2020

HAL is a multi-disciplinary open access archive for the deposit and dissemination of scientific research documents, whether they are published or not. The documents may come from teaching and research institutions in France or abroad, or from public or private research centers.
L'archive ouverte pluridisciplinaire HAL, est destinée au dépôt et à la diffusion de documents scientifiques de niveau recherche, publiés ou non, émanant des établissements d'enseignement et de recherche français ou étrangers, des laboratoires publics ou privés. 


\section{De l'histoire des concepts à l'histoire linguistique des usages conceptuels}

Jacques Guilhaumou, «De l'histoire des concepts à l'histoire linguistique des usages conceptuels », Genèses, 38, 2000, p.105-118.

\section{Introduction}

L'histoire des concepts associée au «tournant linguistique » n'a pas cessé de prendre de l'importance dans le monde germanique et anglophone ces vingt dernières années. Jusqu'à une date récente, les chercheurs français sont demeurés plutôt à distance d'un tel mouvement de redéfinition « langagière » de l'histoire intellectuelle ${ }^{1}$. Il est vrai que ces chercheurs, à la différence de leurs collègues allemands $^{2}$, ont tendance à dramatiser le problème de l'impact du «linguistic turn » dans la recherche historique. Focalisant leur attention sur «le tournant linguistique » à l'américaine d'Hayden White qui semble ne voir dans le discours de l'historien qu'une expression parmi d'autres de l'invention fictionnelle ${ }^{3}$, ils craignent son pouvoir de subversion des règles du métier d'historien. Cependant l'histoire des concepts n'est concernée que marginalement par ce débat, dans la mesure où elle s'interroge en permanence sur le rôle du contexte dans ses modes de compréhension et d'explication des phénomènes conceptuels ${ }^{4}$.

Cette spécificité française, surtout attestée dans le domaine de l'histoire contemporaine, tient également au fait qu'il existe en France des orientations tout à

\footnotetext{
${ }^{1}$ L'ampleur de ce mouvement au cours des années 80 est précisé par Geoff Elgey dans « De l'histoire sociale au 'tournant linguistique' dans l'historiographie anglo-américaine des années 1980 », Genèses №7, 1992. Nous ne rvenons pas sur les très nombreuses références bibliographiques contenues dans cet article, qui concernent un champ d'études, en particulier du côté des historiens sociaux, culturels et intellectuels, beaucoup plus large que l'histoire des concepts.

${ }^{2}$ Voir la critique par Peter Schöttler, dans «Wer hat Angst vor dem 'linguistic turn' ? », Geschichte und Gesellchaft, 23, 1997, de l'analyse faite du « linguistic turn » par Gérard Noiriel dans Sur la 'crise' de l'histoire, Paris, Belin, 1996. A vrai dire, Gérard Noiriel s'en prend plus aux assertions des partisans du «tournant linguistique » sur «l'évanouissement » de la réalité au profit du langage qu'aux résultats des recherches empiriques. Le problème de la connexion empirique entre réalité et discours structure actuellement, nous le verrons, l'enjeu epistémologique de l'histoire des concepts.

${ }^{3}$ La référence principale est l'ouvrage d'Hayden White, Metahistory. The Historical Imagination in NineteenthCentury Europe, Baltimore et Londres, The John Hopkins University Press, dont Roger Chartier fait une minutieuse critique dans Au bord de la falaise. L'histoire entre certitudes et inquiétudes, Paris, Albin Michel, 1998, p.108-125.

${ }^{4}$ Voir sur ce point l'importante mise au point de Mark Bevir, The Logic of the History of Ideas, Cambrige University Press, 1999, qui s'appuie sur une connaissance étendue du champ anglophone de l'histoire des concepts.
} 
fait distinctes en matière d'étude des concepts socio-politiques, et de surcroît une absence de consensus sur le rôle du langage dans la formation des concepts ${ }^{5}$. Pour sa part, l'histoire conceptuelle du politique inscrite dans le lignée de l'oeuvre de François Furet, surtout présente autour de Pierre Rosanvallon ${ }^{6}$ et de la revue $L e$ Débat, étudie la formation et l'évolution des rationalités politiques sur la base d'une description des systèmes de représentation qui les structurent. Ici l'histoire des représentations langagières n'est qu'un aspect de l'histoire des représentations d'une société sur elle-même et n'a donc pas de valeur méthodologique particulière. A l'inverse, l'histoire du discours et la socio-histoire du politique, initiées successivement par Régine Robin ${ }^{7}$ et Maurice Tournier ${ }^{8}$, puis largement reconnues par la présentation de leurs résultats dans la revue Mots, nous ont permis d'associer l'analyse de discours du côté de l'histoire au « tournant linguistique »". Cependant, $\mathrm{du}$ fait de l'importance accordée aux méthodologies linguistiques et lexicométriques, l'analyse de discours et la lexicologie historique éprouvent parfois des difficultés à accorder un statut social aux pratiques langagières, au-delà des considérations sur les stratégies discursives et de la focalisation sur les faits d'étymologie sociale. Reste enfin une socio-histoire du langage conceptuel encore peu répandue ${ }^{10}$, mais qui s'efforce, avec la revue Genèses, de montrer que l'analyse de discours et l'histoire des représentations peuvent s'associer dans une perspective d'histoire sociale. L'accent est mis en dernier lieu sur les déterminations pratiques qui pèsent sur la construction des significations, sans nullement négliger les ressources propres du langage des acteurs producteurs de ces significations.

Ainsi des rapprochements, encore peu perceptibles pour beaucoup de chercheurs français, se précisent depuis quelques années, grâce à cette évolution sensible vers le «tournant langagier » et au détour de rencontres entre chercheurs

\footnotetext{
${ }^{5}$ Voir sur ce point la mise au point de Gérard Noiriel dans Qu'est-ce que l'histoire contemporaine?, Paris, Hachette, 1998 , en particulier pages 179 et svtes.

${ }^{6}$ Le sacre du citoyen, Paris, Gallimard, 1992 et Le peuple introuvable, Paris, Gallimard, 1998.

${ }^{7}$ Dans son ouvrage pionnier sur Histoire et linguistique, Paris, Armand Colin, 1973, dont nous avons eu la chance de suivre la genèse, étudiant alors en maîtrise d'histoire avec Régine Robin.

${ }^{8}$ Voir en particulier son dernier ouvrage Des mots en politique, Paris, Klincksieck, 1997.

9 Dans «A propos de l'analyse de discours: les historiens et le 'tournant linguistique' », Langage \& Société, septembre $1993, \mathrm{~N}^{\circ} 65$

${ }^{10}$ Voir par exemple l'étude de Gérard Noiriel « Socio-histoire d'un concept. Les usages du mot «nationalité » au XIXème siècle », Genèses, 20, 1995.
} 
allemands, anglais, américains, danois, français, finlandais et hollandais, étendues peu à peu à l'ensemble des chercheurs européens soucieux de trouver un terrain commun d'entente en matière d'histoire des concepts socio-politiques. Les réunions récentes à Londres (juin 1998), puis à Paris (octobre 1999) d'une partie des membres du réseau « History of concepts » qui dispose d'une Newsletter et de possibilités de publication dans la revue The Finnish Yearbook of Political Thought ${ }^{11}$, en constitue la manifestation la plus évidente.

Il convient donc d'analyser les tenants et les aboutissants d'une telle rencontre, tout en marquant la spécificité de l'orientation très récente d'historiens linguistes français vers l'histoire conceptuelle sur la base de leur tradition propre. A ce titre, nous devons d'abord préciser comment nous définissons et appréhendons l'histoire des concepts, au regard des débats effectifs et des traductions disponibles en français, certes encore peu nombreux.

De notre point de vue, il s'agit d'un domaine de recherche qui s'intéresse au contexte historique de signification des concepts majeurs du vocabulaire sociopolitique, sans pour autant le réduire à de simples considérations lexicologiques dans la mesure où ces concepts sont appréhendés au sein de relations sémantiques multiples et plus largement d'une interaction sociale à caractère langagier. L'histoire des concepts prend donc en compte les usages d'une langage spécifique dans une situation spécifique à l'intérieur desquelles les concepts sont développés par des auteurs, des acteurs et des orateurs spécifiques.

\footnotetext{
${ }^{11}$ Les volumes 1 (1997) et 3 (1999) de cette revue publiée par l’Université de Jyväskylä, sous la responsabilité de Kari Palonen, l'un des responsables du réseau, présentent un ensemble de débats autour des travaux de Reinhart Koselleck et Quentin Skinner, et en leur présence, particulièrement intéressants. Les autre membres du comité d'organisation du réseau sont Pim der Boer (Amsterdam), Jan Ifversen (Aarhus), Raymonde Monnier (Paris), Melvin Richter (New-York), Praticia Springborg (Sydney). « History of Concepts Newsletter » est éditée par K. Tilmans, W. Velema et F. Sierhuis à Amsterdam.
} 


\section{I- Aperçu sur l'histoire des concepts.}

Deux courants dominent actuellement l'ensemble du champ, l'histoire sémantique autour de Reinhart Koselleck et l'histoire du discours autour de Quentin Skinner ${ }^{12}$. Nous en proposons une caractérisation succincte dans la mesure où leur présentation est au centre actuellement d'un nombre grandissant de publications en anglais, allemand et italien ${ }^{13}$.

\section{A- La Begriffsgeschichte revisitée.}

Le premier courant se situe en Allemagne, autour de Reinhart Koselleck et de la Begriffsgeschichte, auteur majeur dont plusieurs ouvrages ont été traduits en français ${ }^{14}$. Ce chercheur de réputation internationale, en assurant la direction intellectuelle de la vaste entreprise menée à bien du Geschichtliche Grundbegriffe: Historisches Lexikon zur politisch-sozialer Sprache in Deutschland (Les concepts fondamentaux en histoire: Dictionnaire historique du langage politique et social en Allemagne), en collaboration avec Otto Brunner et Werner Conze ${ }^{15}$, a mis au point une méthodologie spécifique, la sémantique historique des concepts qui s'est amplifié et diversifiée jusqu'à nos jours ${ }^{16}$

Prenant en compte les ressources réflexives des «grands auteurs », leurs horizons de pensée et les champs d'expérimentation de leurs discours par les acteurs qui s'en inspirent, Koselleck met tout particulièrement l'accent sur la

\footnotetext{
${ }^{12}$ L'étude comparative de ces deux courants de recherche par Melvin Richter dans The History of Political ans Social Concepts. A Critical Introduction, Oxford University Press, 1995 est à l'origine de la formation du réseau «History of concepts ». Voir également la série d'échanges entre Pocock, Skinner et Richter dans The Meaning of Historical Termes and Concepts. New Studies on Begriffsgeschichte, H. Lehmann and M. Richter eds., German Historical Institute, Washington DC, Occasional Paper $\mathrm{N}^{\circ} 15$. Kari Palonen propose également une comparaison très précise des points de vue de Koselleck et Skinner dans «Rhetorical and Temporal Perspectives on Conceptual Change », Finnish Yearbook of Political Thought, $\mathrm{N}^{\circ} 3$ (1999).

${ }^{13}$ Nous devons ainsi à Sandro Chignola toute une série de publications sur l'histoire des concepts associée à sa réception en Italie dans des revues telles que Filosofia politica (1/1997 et 3/1999) et Il Pensiero politico (à paraître). Il n'est guère possible de faire quelque chose de comparable dans le cas français.

${ }^{14}$ En particulierVergangene Zukunft. Zur Semantik geschichtliche Zeiten, Frankfurt am Main, Suhrkamp, 1979, et dans sa traduction française, Le futur passé. Contribution à la sémantique des temps historiques, Paris, EHESS, 1990, ainsi que le recueil d'articles, L'expérience de l'histoire, Paris, Le Seuil/Gallimard, 1997.

${ }^{15}$ Geschichtliche Grundbegriffe. Historisches Lexikon zur politisch-sozialer Sprache in Deutschland, Stuttgart, Klett Cotta (1972-).

${ }^{16}$ La mise au point la plus récente sur les nombreux débats autour de cette vaste entreprise est celle Hans Erich Bödeker, «Concept-Meaning-Discourse, Begriffsgeschichte Reconsidered », in Iain Hampster-Monk, Karin Tilmans and Frank Van Vree, History of Concepts: comparative Perspectives, Amsterdam University Press, 1998.
} 
période 1750-1850, désignée par l'expression de Sattelzeit, en matière de changement $\mathrm{du}$ langage politique et social. Travail néologique, nouvelles expressions, politisation des concepts, temporalisation de l'histoire, mise en argument des concepts dans le mouvement historique, réalisations d'attentes dans des expérimentations conceptuelles localisées, etc. s'entrecroisent au cours de cette période de changement fondamental pour lui donner un caractère de rupture sur la longue durée prise en compte dans cette entreprise dictionnairique.

Considérant que «définir ce que représente le temps de l'histoire est, de toutes les questions posées par science historique, l'un des plus difficiles à résoudre ${ }^{17}$, Koselleck positionne le problème de la temporalisation des concepts ${ }^{18}$ au centre de l'entreprise collective qu'il impulse. C'est bien au cours de la seconde moitié du 18ème siècle qu'une nouvelle expérience du temps historique s'impose en Europe. La référence à un temps propre devient un élément central de l'usage et de la signification des concepts. L'immanence du temps s'incarne alors dans une forme linguistique, «L'Histoire », qui tend à devenir un concept réflexif, donc indépendant d'un objet et/ou d'un sujet précis. D'autres concepts apparaissent, en particulier ceux de «mouvement» et de «révolution» («un concept de perspective relevant de la philosophie de l'histoire» précise Koselleck), et constituent une trame historique dans laquelle s'installe une tension temporelle entre le champ d'expérimentation de l'action humaine et son horizon d'attente. Koselleck en conclut que la temporalisation des concepts s'étend non seulement aux concepts qui thématisent le temps historique, mais aussi aux concepts sociopolitiques qui marquent la volonté et la possibilité d'un changement, par exemple ceux de "progrès » et d' «émancipation», «concepts d'avenir » à valeur d'orientation du mouvement historique où la distance temporelle entre le contenu d'expérience et l'horizon d'attente est maximale.

Cependant l'absence de considérations sur les séries imprimées (journaux, pamphlets, autobiographies, etc.) et la limitation des considérations linguistiques au champ de «l'histoire sémantique » devaient inciter des chercheurs proches de Koselleck à proposer une nouvelle entreprise dictionnairique, toujours en cours, le Handbuch politish-sozialer Grundbegriffe in Frankreich, 1680-1820 ${ }^{19}$ (Manuel des concepts politiques et sociaux fondamentaux en France de 1680 à 1820), sous

\footnotetext{
${ }^{17}$ Le futur passé..., op. cit. , p. 9.

18 Cf. «The Temporalization of Concepts», Finnish Yearbook of Political Thought, V. 1, 1997 et «Begriffsgeschichtliche Anmerkung zur Zeitgeschichte», in V. Conxenius, M. Greshat und H. Kocher hrsg, Die Zeit nach 1945 als Thema kirchlicher Zeitgeschichte, Göttingen, Vandenhoek \& Ruprecht, 1988.

${ }^{19}$ München, Oldenbourg, Heft 1-20.
} 
la direction de Hans-Jürgen Lüsebrink, Rolf Reichardt et Eberhard Schmitt. A notre plus grand profit, cette entreprise a noué un dialogue permanent ${ }^{20}$ avec des courants français de recherche, tels que l'histoire sociale des mentalités, surtout dans sa dimension iconique (Michel Vovelle), l'histoire des savoirs et des représentations (Roger Chartier), l'analyse du discours du côté de l'histoire (Jacques Guilhaumou, Régine Robin) et la lexicométrie politique (Maurice Tournier). Roger Chartier et nous-même, nous avons publié dans ce Manuel ${ }^{21}$, s'il fallait souligner plus sa proximité avec les préoccupations méthodologiques des chercheurs français.

Ainsi, l'accent est mis sur une histoire pragmatique et culturelle des concepts qui associe de plus en plus les sources iconographiques aux textes. A ce titre, les concepts ne sont plus appréhendés uniquement dans leur résonance abstraite, mais aussi dans leur dimension corporelle et leur charge émotionnelle sur la base des sources visuelles. La dimension réflexive des concepts dans leur historicité même s'en trouve renforcée: il ne s'agit donc pas de construire une sémiologie du concept, mais d'en appréhender la vision que les contemporains en ont par leur conscience collective sur la base d'une combinaison d'éléments verbaux et iconiques. Le « jeu de la Révolution française », héritier du jeu de l'oie dans sa forme, matérialise, en isolant des termes singuliers par des représentations visuelles, les nouveaux concepts-clés de l'humanité agissante. Point de vue synthétique, il éclaire l'entreprise du Handbuch sous un jour particulier: son objectif n'est pas de proposer une standardisation pragmatique du format Dictionnaire, mais de rendre visible une dynamique conceptuelle diversifiée où l'omniprésence de l'ambiguïté, de l'équivoque et de jeux antithétiques interdisent toute construction conceptuelle statique ${ }^{22}$. Ainsi chaque fascicule comprend l'analyse de plusieurs entrées lexicales sans lien alphabétique, mais situées à l'horizon d'une mise en réseau des concepts dans un schème conceptuel général, métaphorisé par les cases du jeu révolutionnaire de l'oie.

\footnotetext{
${ }^{20}$ Voir les mises au point de Peter Schöttler, « Sozialgeschichtliches Paradigma und historische Diskursanalyse », in Diskurstheorien und Literaturwissenschaft, hrsg v. J. Fohrmann und H. Müller, Frankfurt am Main, 1988 et de Rolf Reichardt, "Historische Semantik zwischen lexicométrie und New Cultural History », in Aufklärung und Historische Semantik, R. Reichardt hrsg, Berlin, Duncker \& Humblot qui fournissent un nombre impressionnant de références bibliographiques dans les domaines concernés par leur propos.

${ }^{21}$ Respectivement «Civilité », Handbuch politish-sozialer Grundbegriffe in Frankreich, 1680-1820, op. cit., Heft 4, 1986 et « Subsistances (pain, bleds, grains) », Heft 19-20, 1999.

${ }^{22}$ Voir la mise au point la plus récente de Rolf Reichardt dans « Historical Semantics and Political Iconography: The Case ot the French Revolution (1791-1792) », in I. Hampster-Monk , K. Tilmans and F. Van Vree, History of Concepts: comparative Perspectives, Amsterdam University Press, 1998.
} 
Le Handbuch, Dictionnaire de sémantique historique, pose donc la question de la pertinence du choix du «format lexical ${ }^{23}$ : dans quelle mesure un inventaire alphabétique peut-il permettre de configurer significativement un vocabulaireressource du langage politico-social à un moment donné ? De fait, le choix d'une période plus courte, le 18ème siècle français, fortement marquée par des phénomènes de rupture discursive permet de mieux cibler le moment historique et son continuum mémoriel. Il s'avère ainsi complémentaire d'un travail monographique, comme le démontre l'étude exemplaire de Han-Jürgen Lüsebrink et Rolf Reichardt sur la «Prise de la Bastille ${ }^{24}$. Enfin, ces deux auteurs se sont également proposés d'étendre l'histoire des concepts au champ des transferts culturels pour la période $1770-1820^{25}$. L'enquête collective qu'ils ont ainsi impulsée sur une vaste base archivistique, et en complément du Handbuch, permet désormais d'appréhender avec exactitude les espaces du transfert des nouveaux concepts socio-politiques de la France vers l'Allemagne ${ }^{26}$. C'est ainsi que nous pouvons suivre les voies du transfert et de l'appropriation de la notion de « révolution », élément central de la temporalisation des concepts si chère à Reinhard Koselleck ${ }^{27}$. Au delà des débats qu'elle suscite, l'histoire des concepts en Allemagne relève d'un projet d'ensemble foncièrement unitaire.

\section{B- Des actes de langage au contexte rhétorique: l'école de Cambridge.}

$\mathrm{Du}$ « format lexical » à la monographie, nous débouchons insensiblement sur le second courant majeur de l'histoire des concepts, "l'école de Cambridge » autour de John G. A. Pocock et surtout de Quentin Skinner ${ }^{28}$. Nous sommes ainsi

\footnotetext{
${ }^{23}$ Le débat sur la pertinence du « format lexical », c'est-à-dire sur le choix de la forme dictionnaire est au centre des échanges critiques entre Koselleck et Skinner, ce dernier récusant tout approche de l'histoire des concepts autre que monogaphique. Voir les références des notes 12 et 13.

${ }^{24}$ Die « Bastille ». Zur Symbolgeschichte von Herrschaft und Freiheit, Frankfurt am Main, Fisher, 1990.

${ }^{25}$ Voir la mise en place du projet, et la présentation de premiers résultats dans Hans-Jürgen Lüsebrink et Rolf Reichardt, «Histoire des concepts et transferts culturels, 1770-1815. Note sur une recherche », Genèses, 14, janvier 1994.

${ }^{26}$ Hans-Jürgen Lüsebrink und Rolf Reichardt Hrsg., Kulturtransfer im Epochenumbruch Frankreich-Deutschland 1770 bis 1815, deux volumes, Leipziger Universitätverlag, 1997. Voir aussi Matthias Middel, «La Révolution frnaçaise et l'Allemagne: du paradigme comparatiste à la recherche des transferts culturesl », Annales Historiques de la Révolution française, $\mathrm{N}^{\circ} 317$, juillet-septembre 1999.

${ }^{27}$ Rolf Reichardt, «Die Revolution -, 'ein magisher Spiegel'. Historich-politische Begriffsbildung in französischdeutschen Übersetzungen », ibid., volume 2, p. 883-999.

${ }^{28}$ Une bibliographie des premiers travaux de Quentin Skinner se trouve dans l'ouvrage Meaning and Context: Quentin Skinner and his critics, ed. by James Tully, Oxford/Princeton, 1988. Skinner dirige la collection « Ideas in
} 
confronté à une histoire du discours centrée sur les langages politiques dont l'intérêt quasi-exclusif pour les grands textes n'entache pas la nouveauté méthodologique que Pocock précise dans les termes suivants: "It seems to us that history of political language and discours can better be writen if we focus our attention on the acts of articulation and conceptualisation performed by thinkers as agents in the world of speech, and on the matrice of language and rhetoric within which thez are constrained to speak but which thez modify by the speech-acts they perform ${ }^{29}$.

. En effet il s'agit de montrer, d'une étude à l'autre, que les auteurs considérés ne se contentent pas de conceptualiser dans une situation spécifique, mais en écrivant, ils investissent le contexte dans leur mouvement argumentatif par des actes de langage: ils disent donc quelque chose au sens performatif du dire, ils sont agissants. Dès ses premiers travaux ${ }^{30}$, Skinner se démarque ainsi de l'histoire classique des idées en s'intéressant à l'histoire des concepts dans le contexte d' «actions linguistiques », définies comme le moment où le potentiel normatif des concepts est pris dans l'action politique. Il s'agit bien de prendre en compte l'usage argumentatif de concepts appréhendés dans leur force illocutionnaire, donc en tant qu'actes de langage. Ce qui intéresse Skinner, c'est d'étudier des textes à partir de contextes qui lui permettent d'identifier ce que les auteurs de ces textes ont fait en écrivant comme ils l'ont fait.

Actuellement Skinner s'oriente plutôt vers l'étude du changement conceptuel dans une perspective rhétorique. C'est ainsi qu' il présente l'œuvre de Hobbes ${ }^{31}$ non pas à travers l'exposition statique d'un ensemble de concepts abstraits, mais il décrit les catégories hobiennes au sein de la chaîne argumentative que le contexte contribue à mettre en mouvement. Il analyse donc, dans la lignée des réflexions d'Austin et de Wittgenstein, leur dimension d'action langagière à partir d'une description minutieuse d'un contexte rhétorique, culturel et politique. Ainsi il valorise des lignes d'arguments, et leur mouvement, utilisés par Hobbes luimême pour réactiver tel ou tel élément du contexte avec pour but d'introduire un changement, en particulier stylistique et rhétorique, dans les débats anglais du XVIIème siècle. Il met ainsi l'accent sur la culture rhétorique de l'humanisme

\footnotetext{
Context » à Cambridge University Press qui comprend actuellement plus de quarante titres et anime un programme de recherche sur The Early Modern European Republicanism.

29 «The Machiavellian Moment Revisited: A Study in History and Ideology », Journal of Modern History, 53, march 1981.

${ }^{30}$ Foundations of Modern Political Thought, deux volumes, Cambridge University Press, 1978.

${ }^{31}$ Reason and Rhetoric in the Philosophy of Hobbes, Cambridge University Press, 1996.
} 
renaissant, et la manière dont elle occupe une position contextuelle majeure dans l'évolution de la position de Hobbes en matière de construction d'une "société civile », et donc dans son choix final d'une union intime entre raison et rhétorique.

Cependant le contextualisme de Skinner ne se confond pas avec celui de John Pocock ${ }^{32}$. C'est ainsi que le «moment machiavelien ${ }^{33}$ est identifié à partir de certains vocabulaires conceptuels mis à disposition par les contemporains de la Renaissance pour tenir un discours sur les systèmes politiques et leur changement. Il s'agit alors, à partir de la formation du républicanisme florentin, d'identifier la traduction d'un modèle d'idéal civique de la personnalité dans le langage de l'humanisme civique, véritable paradigme civique opératoire pour près de deux siècles. Les usages de ce langage civique ont donc une valeur heuristique.

Ainsi le point de vue contextualiste de Pocock consiste dans la recherche du sens d'un texte à l'intérieur d'un paradigme langagier déterminé. Par contraste, Skinner est plus spécifiquement conventionaliste dans la mesure où il s'efforce de comprendre le sens d'un texte sur la base d'une reconstitution minutieuse du contexte des débats et des conventions linguistiques dans lesquels ce texte s'inscrit? Il reproche d'ailleurs à Pocock de s'en tenir trop strictement aux traditions langagières, oubliant ainsi de prendre en compte les conventions linguistiques, en particulier rhétoriques, qui sont à l'origine de la force illocutoire propre à dynamiser l'intervention de l'auteur en faveur de l'action publique ${ }^{34}$. Cependant ces deux auteurs anglophones participent d'un renouveau de l'histoire intellectuelle du côté de l'histoire des concepts formulable dans les termes suivants:

«We shall then be able to see how the concepts we still invoke were initially defined, what purpose they were intended to serve, what view of public power they were used tu underpin. This in turn may enable us to acquire a self-conscious understanding of a set of concepts that we now employ unselfconsciously and, to some degree, uncomprenhendingly $»^{35}$.

\footnotetext{
32 Politics, Language and Time, London, Methuen, 1972, et plus particulièrement l'étude sur « Languages and their implications. The Transformation of the Study of Political Thought». Le contextualisme de «l'école de Cambridge », est interrogé sur son choix langagier et ses limites dans l'ouvrage de Mark Bevir, The Logic of the History of Ideas, Cambridge University Press, 1999.

${ }^{33}$ The Machiavellian Moment, Princeton University Press, 1995 (traduction française, Paris, PUF, 1997).

${ }^{34}$ Voir en particulier, Q. Skinner, «Problems in the Analysis of Political Thought », in J. Tully ed., Meaning and Context, Cambridge University Press, 1985.

${ }^{35}$ Q. Skinner, Liberty before Liberalism, Cambridge University Press, 1998, p. 110.
} 
D'autres projets nationaux s'inscrivent dans le domaine de l'histoire conceptuelle, en particulier les projets hollandais et finlandais ${ }^{36}$. Dans le cas hollandais, l'accent est mis sur le mouvement conceptuel attenant à la propagation du langage national dans les Pays-Bas au cours de la seconde moitié du XVIème siècle, sorte de proto-Sattelzeit par son décalage temporel avec la période 17501850 privilégiée par les Allemands. Chronologiquement parlant, l'analyse conceptuelle se situe ici au plus près du moment de l'humanisme civique florentin mis en valeur par Pocock. Mais elle met plutôt l'accent, surtout avec les travaux de Martin van Gelderen ${ }^{37}$ sur la radicalité sémantique de l'usage de nouveaux concepts au moment de la révolte hollandaise, dans la lignée des réflexions de Koselleck. A la lumière de ses travaux, il apparaît que c'est autour de la formation de l'Etat-nation, et de sa crise contemporaine, que se situe là une grande partie de l'interrogation en histoire des concepts ${ }^{38}$.

\section{II - L'histoire linguistique des usages conceptuels}

Nous l'avons déjà dit, il n'existe pas véritablement en France de courants en histoire des concepts aussi nettement constitués qu'en Angleterre et en Allemagne pour s'en tenir à l'espace européen. Cependant, la riche expérience, depuis les années 1970, du laboratoire de lexicologie politique de l'Ecole Normale Supérieure de Saint-Cloud, associé au champ de l'analyse de discours du côté de l'histoire $^{39}$ débouche actuellement sur une histoire linguistique des usages conceptuels dont nous souhaitons présenter les caractéristiques majeures.

\footnotetext{
${ }^{36}$ Pour le cas hollandais voir l'étude de Pim den Boer, « The Historiography of German Begriffsgeschicchte enad the Dutch Project of Conceptual History » dans I. Hampsher-Monk, K. Tilmans, F. Van Vree, History of concepts : comparative perspectives, Amsterdam University Press, 1998. Le cas finlandais, hormis dans la revue déjà citée The Finnish Yearbook of Political Thought, est présenté par Tuija Pulkkinnen dans «One language, one mind. The Nationalist Tradition in Finnish Policial Culture», in Europe's Northern Frontier. Perspectives on Finland's Western Identity, Tuomas M. S. Lehtonen ed., Porvoo, PS-Kustannus, 1999.

${ }^{37}$ The political Thought of the Dutch Revolt (1555-1590), Cambridge University Press, 1992.

${ }^{38}$ Voir sur ce point l'article synthétique, accompagné d'une bibliographie abondante, de Istvan Hont, «The Permanent Crisis of a Divided Mankind: 'Contemporary Crisis of the Nation State' in Historical Perspective », Political Studies, XLII, 1994.

39 Jacques Guilhaumou, Denise Maldidier et Régine Robin, Discours et archive, Liège, Mardaga, 1994 au moment même où sont soutenues les premières thèses en ce domaine, tout particulièrement celles de Sophie Wahnich, L'étranger, paradoxe de l'universel. Analyse du discours politique révolutionnaire sur l'étranger de la Fédération à Thermidor, thèse pour le doctorat d'Etat, Université de Paris I, dir. M. Vovelle, 1994 (L'impossible citoyen. L'étranger dans le discours de la Révolution française, Paris, Albin Michel, 1997) et de Marc Deleplace, La notion d'anarchie pendant la Révolution française (1789-1801). Formation d'un concept, Thèse de Doctorat de l'Université de Paris I, Michel Vovelle dir., 1994. Les langages de la Révolution française y occupent une place privilègiée, et
} 
Deux projets français, emboîtés l'un dans l'autre, concernent donc le champ de l'histoire des concepts. Ils s'inscrivent à mi-chemin de l'enquête monographique et de l'élaboration d'un Dictionnaire des usages socio-politiques du français contemporain.

Le plus ambitieux des deux demeure à l'état de prototype thématique d'un tel Dictionnaire des mots socio-politiques pour l'ensemble de la période contemporaine. L'ouvrage collectif sur In/égalité/s, élaboré sous la direction de Pierre Fiala ${ }^{40}$, en donne les orientations majeures. Il résulte d'un travail transversal à l'ensemble des équipes du Laboratoire "Analyse de corpus, lexicométrie et textes politiques », donc sur la période 18ème-20ème siècles, avec l'apport de la banque de données Frantext (Institut National de la Langue Française) et de la textothèque de ce laboratoire. Il s'agit donc d'un travail sur un corpus de textes qui nous confronte avec une description des usages dans des discours diversifiés et une approche partiellement quantitative selon les méthodes lexicométriques d'analyses lexicales et discursives.

La démarche diachronique sur des corpus très divers part du principe d'une dispersion très forte et de la nature composite du discours socio-politique, qui concerne aussi bien des pratiques langagières décrites tant dans l'archive que dans l'imprimé que des textes de grands auteurs, sans oublier les produits actuels de la communication médiatique. Par ailleurs, le propos des chercheurs français met l'accent sur le manque d'attention, dans l'histoire conceptuelle du politique, pour les propriétés linguistiques des mots, leurs variations morphologiques et sémantiques en-deçà de considérations langagières générales. Nous pouvons alors parler d'un essai de description diachronique d'un terme (et de sa famille morphologique) caractéristique des discours politiques contemporains, en l'occurrence le terme d'égalité.

Reste que la dizaine d'analyses des variations, déplacements et substitutions proposée autour de ce thème demeurent monographiques, et qui plus est ne prennent pas vraiment en compte le contexte au sens large. Il s'agit donc d'un projet limité, tout du moins à travers ce prototype thématique, qui présente essentiellement l'intérêt de nous donner un éventail assez complet des méthodes d'analyse linguistique et discursive sur des corpus diversifiés de discours politiques. La question de la mise en oeuvre d'un Dictionnaire contextuel, qui

font désormais l'objet d'un début de synthèse. Cf. Jacques Guilhaumou, L'avènement des porte-parole de la République (1789-1792). Essai de synthèse sur les langages de la Révolution française, Lille, Presses Universitaires du Septentrion, 1998.

${ }^{40}$ In/égalité/s. Usages lexicaux et variations discursives (18ème-20 ème siècles), Paris, L'Harmattan, 1999. 
allierait l'analyse discursive des concepts, la prise en compte de la diversité des usages et de la multiplicité des pratiques langagières, la description de leurs propriétés linguistiques et de leurs variations historiques à la systématicité lexicale de la démarche dictionnairique demeure ici en suspens. Une autre solution, le Dictionnaire des fréquences, si elle s'applique à de très grands corpus, peut s'avèrer très productive dans une perspective contextualiste sur une base quantitative: elle associe en effet l'analyse du champ lexical d'une notion-concept, de ses spécificités à l'étude de son réseau sémantique. Cependant une seule expérimentation a été effectuée en ce domaine; elle concerne le corpus des résolutions des congrès fédéraux tenus par quatre organisations syndicales, la CGT, la CFDT, FO et la CFTC entre 1971 et $1990^{41}$.

Le second projet, mis en place dans la conjoncture du bicentenaire de la Révolution française, est beaucoup plus resserré chronologiquement, mais se concrétise à travers une série de publications. Il s'agit du Dictionnaire des usages socio-politiques du français (1770-1815), constitué à l'initiative de l'équipe «18ème et Révolution française », dont nous assurons actuellement la direction avec Raymonde Monnier et Marie-France Piguet, et publié dans la collection «Saint-Cloud» dirigée par Annie Geffroy. Six fascicules ont déjà été publiés ${ }^{42}$. Le premier volume est paru en 1985, au même moment que le premier fascicule du Handbuch, sans pour autant vouloir rivaliser avec cette vaste entreprise allemande.

Fruit d'une collaboration entre historiens, lexicologues et linguistes, ce Dictionnaire accorde un place prépondérante à la présentation de résultats discursifs, donc à la recension et l'interprétation d'usages en contexte. S'adressant pour une part à la communauté des historiens de la période révolutionnaire, il ne s'en tient pas au «format lexical », voir refuse toute nomenclature a priori, au profit d'une approche aussi diversifiée que possible des pratiques langagières. Nous sommes donc bien là dans l'espace de l'histoire langagière des concepts, à l'égal de l'histoire des concepts prise dans le "tournant linguistique ». C'est pourquoi la perspective ouverte par ce projet débouche actuellement sur une réflexion autour de ce que nous appelons désormais l'histoire linguistique des usages conceptuels.

\footnotetext{
${ }^{41}$ Anne-Marie Hetzel, Josette Lefèvre, René Mouriaux, Maurice Tournier, Le syndicalisme à mots découverts. Dictionnaire des fréquences (1971-1990), Paris, Syllepse, 1998.

${ }^{42}$ Dictionnaire des usages socio-politiques du français (1770-1815), fascicules 1-6, Paris, Klincksieck, 1985-1999.
} 
D'abord, et en liaison avec les chercheurs allemands soucieux de sémantique historique, de pragmatique historique de texte et de sociolinguistique historique ${ }^{43}$, cette entreprise s'appuie sur un point de vue herméneutique qui considère l'analyse des conditions langagières d'apparition des formes discursives comme un accès privilégié à leur compréhension historique. Dans les termes de l'analyse de discours, une fois inséré, par la lecture d'archives, dans une configuration discursive, l'énoncé ainsi attesté est appréhendé dans sa mise en acte, par la concrétisation d'usages de notions, d'arguments dans un continuum entre le contexte, les ressources et les thèmes du discours socio-politique. L'histoire linguistique des usages conceptuels est donc aussi une histoire pragmatique dans la mesure où elle accorde, à l'exemple de Skinner, une grande importance aux actes de langage constitutifs d'usages performatifs de notions socio-politiques.

Cependant, d'un fascicule à l'autre du Dictionnaire des usages sociopolitiques, nous passons d'un intérêt quasi-exclusif pour l'usage de mots désignants et/ou instituants du discours révolutionnaire dans des conjonctures précises à une ouverture vers des configurations discursives plus diversifiées, par le fait de la lecture d'archives et du recours aux banque de données, et des problématiques langagières plus élaborées, au point de rencontrer des questions proprement langagières de l'histoire des concepts, en particulier celles de l'abus et du pouvoir des mots.

De fait les choix thématiques des six volumes publiés peuvent être regroupés en trois volets. Deux volumes (I-1985 et IV-1989) concernent très classiquement une série de désignants socio-politiques (sans-culottes, aristocrates, anarchistes, etc.) tout à fait courants dans le discours révolutionnaire, mais sujets à des retournements/réélaborations situés ici précisément dans leur contexte discursif. Les désignants socio-politiques concernent ainsi une ressource lexicale peu homogène sémantiquement, mais dont la valeur autodésignante et la forte prise en charge énonciative permettent d'en situer assez précisément le contexte et les conditions d'émergence. Deux autres volumes (III-1998 et V-1991) s'intéressent aux outils linguistiques, essentiellement les Dictionnaires, et à la conscience linguistique de l'époque. Les études présentées, d'une grande diversité méthodologique, s'insèrent dans le champ plus large de l'histoire des idées et des théories linguistiques ${ }^{44}$. Les deux volumes restants (II - 1987 et VI - 1999), parcourent un trajet significatif des notions-concepts aux notions pratiques.

\footnotetext{
${ }^{43}$ Hormis Hans Erich Bödeker, Reinhart Koselleck, Hans-Jürgen Lüsebrink, Rolf Reichardt déjà cités, il s'agit aussi de Brigitte Schlieben-Lange, historienne des idées linguistiques, spécialiste des Idéologues et auteur d'un ouvrage de synthèse sur Idéologie, révolution et uniformité de la langue, Liège, Mardaga, 1996.

${ }^{44}$ Voir l'Histoire des idées linguistiques, dirigée par S. Auroux, Liège, Mardaga, trois volumes, 1989-1999.
} 
Avec les notions-concepts, il s'agissait, dans la conjoncture du bicentenaire de la Révolution française, de restituer, par le retour à l'archive et au contexte discursif, la portée conceptuelle, au sein même des événements révolutionnaires, de notions toujours citées par les historiens, mais particulièrement mal connues dans leur réalité langagière, c'est-à-dire leur mise en argument dans des trajets discursifs. Si le choix de la série lexicale terreur, république, liberté, tyrannie, etc. n'étonne personne, reste que ces notions-concepts constitutives du «nouvel ordre des choses » sont présentement appréhendées plus dans leur émergence discursive que dans leur résonance doctrinale.

Cependant une telle approche discursive des notions organisatrices du discours révolutionnaire prises dans la dynamique contextuelle devait finir par rencontrer les préoccupations de l'histoire des concepts. Ainsi l'accent mis par Koselleck et le Geschichtliche Grundbegriffe sur l'importance grandissante de la notion-concept de démocratie au 18ème siècle se retrouve dans l'analyse historicolinguistique de Raymonde Monnier, plus centrée sur la période révolutionnaire ${ }^{45}$. A l'articulation de l'horizon d'une forme idéale et de l'expérimentation de la dynamique démocratique, un projet civique, donc en appui sur l'opinion publique, confère à la société sa temporalité propre.

A ce titre, le dernier volume publié sur les notions-pratiques permet de caractériser le champ discursif de notions mises en acte dans un étroite relation aux objets et aux circonstances. Nous retrouvons des notions (humanité, loi, harmonie etc.) héritées de la tradition des Lumières, mais dont la diversification dans l'événement même leur donne une dimension pratique définissable à partir des seules ressources textuelles attestées. Nous entrons dans l'univers de la rationalité de l'action, de «l'action qui convient » au sens sociologique ${ }^{46}$.. Ainsi le champ sémantique d'une notion-pratique ne peut rendre compte de sa valeur conceptuelle dans le seul cheminement du mot, elle doit considérer une diversité d'usages de termes appréhendés dans les mêmes circonstances, proches du même objet, donc présent dans les mêmes actions. Nous nous rapprochons ainsi singulièrement de l'histoire sémantique, à la différence près d'une attention prononcée à l'action qui convient. En effet, dans une perspective proche de l'ethnométhodologie ${ }^{47}$, mais

\footnotetext{
${ }^{45}$ R. Koselleck, «The Temporalization of concepts', op. cit.; « Demokratie », Geschichtliche Grundbegriffe, volume 1, op. cit.; R. Monnier, « Démocratie et Révolution française », Mots N59, juin 1999.
46 L'histoire des concepts s'intéresse à l'histoire des notions en acte. Aussi elle prend en compte les raisons pratiques. Voir en particulier Laurent Thévenot, «L'action qui convient», Les formes de l'action. Sémantique et sociologie, Raisons pratiques, 1, Paris, Editions de l'EHESS, 1990.

${ }^{47}$ Ce que les ethnométhodologues appellent «la réflexivité des descriptions sociales », c'est-à-dire le fait qu'un langage descriptif d'un membre d'une société est à la fois une activité sociale pratique et une activité de connaissance concertée, a fortement influencé les recherches en histoire du discours sur archives depuis les années 80. Sur la
} 
étendue du discours ordinaire au discours socio-politique, il est désormais possible de rendre compte de la manière dont les notions s'organisent en faisant réflexivement sens dans leur contexte propre, qui structure en outre les expérimentations et les attentes des acteurs concernés par l'usage de ces notionspratiques. Nous sommes ainsi projeté dans le monde des accomplissements notionnels pratiques qui se réalisent au sein même des processus constitués par les membres d'une société.

\section{III - Conclusion : sur la connexion empirique entre la réalité et le discours.}

En fin de compte, au terme de ce trajet notionnel, il apparaît qu'une notionconcept est attestée dans une situation précise d'échange des ressources disponibles pour la rendre intelligible. La médiation des observateurs, spectateurs et acteurs dans l'argumentaire de cette notion, et de sa valeur réflexive, introduit un co-partage de la responsabilité de l'usage des mots en société, et donc une volonté d'en clarifier l'emploi social. La question du contrôle sémiotique de «l'abus des mots » devient ainsi un véritable enjeu méthodologique ${ }^{48}$.

«L'abus des mots » comme critère méthodologique en matière d'usages et de controverses conceptuels nous renvoie par ailleurs au problème crucial du rapport de la réalité au discours. C'est d'ailleurs là un des rares endroits où le débat se soit instauré entre Reinhard Koselleck et un chercheur français, en l'occurrence nousmême, à l'occasion du colloque de Bielefeld en $1985^{49}$. Ce court échange, Koselleck devait l'amplifier par la suite dans son étude sur " Histoire sociale et histoire des concepts» ${ }^{50}$ où il qualifie l'histoire du discours en France d' " histoire linguistique », dénomination que nous avons reprise à notre compte dans la mesure

perspective proprement langaggière, voir Jean Widmer, Langage et action sociale. Aspects philosophiques et sémiotiques du langage dans la perspective de l'ethnométhodologie, Suisse, Editions Universitaires de Fribourg, 1986.

${ }^{48}$ La question de l'apport méthodologique du débat autour de l'abus des mots est mise en avant dès 1985 dans le premier fascicule du Handbuch politish-sozialer Grundbegriffe in Frankreich, 1680-1820, op. cit, par Rolf Reichardt, puis a été l'un des thèmes de discussion de la réunion 1999 du réseau « History of concepts ».

Rolf Reichardt, Schmitt Eberhard hrsg., Die Französischen Revolution als Bruch des geselleschaftlichen Bewusstseins, München, Oldenbourg, 1988. Notre intervention dans un des débats a porté sur le thème «Die Reflexivität des sozialen Bewusstseins: von den Archiven zum Begriff », s.666-668, et la réponse de Koselleck sur «Probleme der Relationbestimmung der Texte zur revolutionären Wirklichkeit », s. 664-666.

50 «Sozialgeschichte und Begriffsgeschichte », in W. Cheider und V. Sellin hrsg., Sozialgeschichte in Deutschland, Göttingen, Vandenhoeck \& Ruprecht, 1986, vol. 1. Texte traduit dans L'expérience de l'histoire, op. cit. 
où elle marque bien, dans l'optique de l'histoire des concepts, la différence avec l' « histoire sémantique »,

A l'époque, soucieux de minimiser le poids des interprétations historiographiques courantes de la Révolution française, nous préconisions une position herméneutique "forte » au titre d'une réflexivité générale des discours, à vrai dire sous l'influence de Gadamer ${ }^{51}$, mais jugée alors extrême en matière d'interprétation des textes, surtout lorsque nous affirmions que le contexte n'est qu'une des ressources du texte. Koselleck nous mettait alors en garde contre les dangers de ce qu'il percevait comme du pantextualisme: d'une part faire l'impasse sur le statut cognitif des concepts qui contribue à leur connaissance scientifique, d'autre part, plus grave encore, dénier la connexion empirique entre la réalité et le discours par le constat unilatéral de la perfomativité généralisée des discours. Depuis lors, plus sensible à la dimension référentielle de l'événement langagier, nous admettons sans mal l'existence d'une réalité hors du discours ${ }^{52}$, tout en restant très attaché à une attitude herméneutique face aux énoncés d'archive, dans la lignée des travaux de Michel Foucault. Qui plus est, Koselleck lui-même précise que l'affirmation de la différence entre la réalité et le langage part du constat d'un écart entre l'histoire en cours et les conditions de possibilité langagières: "Une histoire ne s'accomplit pas sans paroles, mais elle ne se confond jamais avec elles, n'y est pas réductible ». C'est dire aussi que la connaissance des conditions de possibilité langagières est indispensable pour accéder à la connaissance historique, sans pour autant se réduire à cette connaissance, donc qu'il convient de décrire les conditions langagières de formation de la réalité pour pouvoir en restituer l'intelligibilité. De même Skinner, en contextualiste convaincu, met certes l'accent sur la réalité du contexte, la décrit minutieusement, mais montre, dans le même mouvement, que ce contexte n'est véritablement intelligible que dans sa mise en acte au sein des arguments textuels. Avec le cas hobbien déjà abordé ${ }^{53}$, il consacre

\footnotetext{
${ }^{51}$ En particulier lorsqu'il écrit que l'art de comprendre procède du moment où « la contemporanéité du sens qui se déclare dans le texte se produit grace à son interprétation (quand elle réussit) » à la différence de l'entreprise historienne qui procède d'une construction continuelle de sens dans le but de trouver un sens caché. Cette réflexion est contenue dans un article de 1985, "Zwischen Phänomenologie und Dialektik», et traduit dans L'art de comprendre. Ecrits II, Paris, Aubier, 1991, p.33.

52 Voir à ce propos l'ouvrage de John R. Searle, The Construction of Social Reality, New York, Free Press, 1995
(traduction française, Paris, Galllimard, 1998). Théoricien des actes de langage, il argumente sur le fait que le monde
existe indépendamment de nos représentations, tout montrant que l'élément linguistique est constitutif de la réalité
institutionnelle. Ce réalisme externe, ontologique présuppose un lien entre l'arrière-plan cognitif et le monde des
énoncés. Cette connexion réaliste est la condition de l'intelligibilité des énoncés, y compris dans leur dimension
performative.

${ }^{53}$ Reason and Rhetoric in the Philosophy of Hobbes, Cambridge University Press, 1996.
} 
ainsi une grande partie de son étude à la caractérisation du contexte de l'émergence d'une «science civile» dans l'oeuvre de Hobbes, plus précisément à la réélaboration de la culture rhétorique de l'humanisme civique de la Renaissance au sein de la pensée anglaise du 17 ème siècle. Puis il s'intéresse au processus de redescription rhétorique, à partir d'une telle base contextuelle, au sein même du style et de la stratégie argumentative de Hobbes. C'est ainsi qu'il met en évidence des effets rhétoriques susceptibles de rendre plus persuasif les nouveaux arguments de la «science civile », donc de contribuer à lutter en quelque sorte contre « l'abus des mots ». La mise en argument du contexte rhétorique permet donc non seulement de valoriser les vérités rationnelles par des règles d'usage partagées au sein de la communauté des citoyens, mais aussi d'orienter le lecteur vers une position active en tant que spectateur engagé par la force rhétorique des arguments sur la voie des vertus publiques, donc contre la corruption morale du langage.

Le cas français du 18ème siècle avec l'institution rationnelle de la langue française comme langue commune, en particulier dans le cadre du Dictionnaire de l'Académie, puis comme langue nationale pendant la Révolution française contre «l'abus des mots » ouvre une perspective méthodologique proche en histoire des concepts, mais en association avec l'histoire des événements linguistiques ${ }^{54}$. Un sujet politique de la langue s'impose progressivement au moment même où se constitue un nouvel espace public sur la base de jugements et d'arguments spécifiques. En fin de siècle, la position du spectateur dans un tel acte de juger devient essentielle à l'établissement d'un consensus en faveur de l'idée émancipatrice de droit, comme l'a si fortement souligné Kant ${ }^{55}$.

Finalement, l'histoire des concepts admet l'existence de lignes de résistance du réel à toute interprétation purement textuelle, voire fictionnelle, de la réalité. Mais il s'agit de lignes de tendance du réel toujours susceptibles d'être négociées par des auteurs, des acteurs, des orateurs et des spectateurs qui disposent de possibilités langagières ayant valeur de ressources interprétatives diversifiées, donc suffisantes pour comprendre le réel. Ainsi se dégage un souci commun des divers courants de l'histoire conceptuelle, y compris l'histoire linguistique des usages conceptuels, de conserver la connexion empirique entre l'action concrète et le discours. Nous sommes loin, nous semble-t-il, des inquiétudes des historiens

\footnotetext{
${ }^{54}$ A propos de cette forte spécificité linguistique de l'histoire du discours en France, voir notre réflexion générale sur "Vers une histoire des événements linguistiques. Un nouveau protocole d'accord entre l'historien et le linguiste », Histoire/Epistémologie/Langage, décembre 1996. Nous préparons actuellement une étude sur l'histoire des événements linguistiques de «La Langue Française » au 18ème siècle.

${ }^{55}$ C'est à ce titre que nous proposons d'insérer certains aspects de l'histoire des concepts dans une esthétique de l'événement, en qualifiant plus précisément son contexte langagier sur la base de l'étude des événements linguistiques. Sur le rapport à Kant, voir notre ouvrage La parole des sans. Les mouvements actuels à l'épreuve de la Révolution française, Paris, ENSéditions, 1998, p. 108-118.
} 
français sur les risques, en adoptant le «tournant linguistique », de détruite le régime de vérité propre au métier d'historien. Les développements actuels de l'histoire des concepts, tout particulièrement en Europe, devraient donc lever les obstacles à son introduction dans le champ français de la recherche. 